\title{
COMPUTATION OF GREEN'S MATRICES FOR BOUNDARY VALUE PROBLEMS ASSOCIATED WITH A PAIR OF MIXED LINEAR REGULAR ORDINARY DIFFERENTIAL OPERATORS
}

\author{
T. GNANA BHASKAR and M. VENKATESULU \\ Department of Mathematics \\ Sri Sathya Sai Institute of Higher Learning \\ Prasanthinilayam, 515134 INDIA
}

(Received July 29, 1991 and in revised form January 15, 1992)

\begin{abstract}
An algorithm for the computation of Green's matrices for boundary value problems associated with a pair of mixed linear regular ordinary differential operators is presented and two examples from the studies of acoustic waveguides in ocean and transverse vibrations in nonhomogeneous strings are discussed.
\end{abstract}

KEY WORDS AND PHRASES: Nonexplicitly mixed, matchingly mixed, boundary value problem, Green's matrices.

1991 AMS SUBJECT CLASSIFICATION CODES: 34XX, 34BXX, 34B27

\section{INTRODUCTION}

Recently, a new class of problems of the type where different differential operators are defined over two adjacent intervals, involving certain mixed (interface) conditions are studied in [1,2,3,4]. These problems involve a pair of differential operators of the type $\tau_{1} u_{1}-\sum_{k=0}^{n} P_{k} D^{k} u_{1}-\lambda u_{1}$, defined on the interval $J_{1}=[a, b]$ and $\tau_{2} u_{2}=\sum_{k=0}^{m} Q_{k} D^{k} u_{2}=\lambda u_{2}$ defined on the adjacent interval $J_{2}=[b, c]$ $-\infty<a<b<+\infty$, where $\lambda$ is an unknown constant (eigenvalue) and the functions $u_{1}$ and $u_{2}$ are required to satisfy certain mixed conditions at the interface $x=b$. In most of the cases, the complete set of physical conditions on the system gives rise to selfadjoint eigenvalue problems associated with the pair $\left(\tau_{1}, \tau_{2}\right)$. Based on the interface conditions these problems can be classified into three types, namely (i) where the values of $u_{1}$ and $u_{2}$ are not explicitly related to each other at $x-b$, (ii) where $u_{1}$ and $u_{2}$ are required to satisfy the continuity conditions at $x=b$, and (iii) where $u_{1}$ and $u_{2}$ satisfy certain matching conditions at $x=b$.

The methods presented in [4] for the construction of Green's matrices for the boundary value problems (BVPs) associated with $\left(\tau_{1}, \tau_{2}\right)$ are theoretical in nature and involve lengthy calculations. Here, in this paper we present (i) simpler algorithms for the computation of Green's matrices for the BVPs associated with $\left(\tau_{1}, \tau_{2}\right)$, and (ii) construct the Green's matrices for the problems found in some physical situations.

Before indicating the division of the work into sections, we introduce a few notations and make some assumptions. For any compact interval $J$ of $R$ and for a nonnegative integer $k$, let $C^{k}(J)$ denote the space of all $k$-times continuously differentiable complex valued functions defined on $J$. For a function $u$, let $u^{(j)}$ denote the $j^{\text {th }}$ derivative of $u$, if it exists. For a compact interval $J$ of $R$ and for a positive continuous (weight) function $r(x)$ defined on $J$, let $L(J, r)$ denote the Hilbert space of all 
Lebesgue measurable complex valued functions $u$ defined on $J$ such that $r(x)|u(x)|$ is integrable over $J$. The inner product in $L(J, r)$ is given by $\langle u, v\rangle-\int u(x) \overline{v(x)} r(x) d x, u, v \in L(J, r)$, where $\overline{v(x)}$ denotes the complex conjugate of $v(x)$, and the norm is given by

$$
\|u\|=\left(\int r(x)|u(x)|^{2} d x\right)^{1 / 2}, \quad u \in L^{2}(J, r) .
$$

Let $H^{k}(J, r)$ denote those functions in $A C^{k}(J)$ such that both $u$ and $u^{(k)}$ are in $L^{2}(J, r)$. Let $C^{k}$ denotes the $k$-dimensional complex space whose elements we take to be column vectors. For $k_{1} \times k_{2}$ matrix $A$ with complex entries, $A^{*}$ denote the $k_{2} \times k_{1}$ matrix which is the conjugate transpose of $A$. Let $A^{-1}$ denote the inverse of a square matrix $A$, if it exists. If $V_{1}$ and $V_{2}$ are vector spaces (over the same field), then $V_{1} \times V_{2}$ denotes the cartesian product of $V_{1}$ and $V_{2}$ taken in that order. For an operator $T$, $D(T), R(T), N(T), \eta(T)$ denote the domain, range, null space and the dimension of the null space of $T$, respectively. Let $X=L\left(J_{1}, r_{1}\right) \times L\left(J_{2}, r_{2}\right)$ be the cartesian product Hilbert space equipped with the inner product $\langle\cdot\rangle$ and the norm $\|\cdot\|$ given by

$$
\left\langle\left\{u_{1}, u_{2}\right\},\left\{v_{1}, v_{2}\right\}\right\rangle=\left\langle u_{1}, v_{1}\right\rangle+\left\langle u_{2}, v_{2}\right\rangle,\left\{u_{1}, u_{2}\right\},\left\{v_{1}, v_{2}\right\} \in X,
$$

and

$$
\left\|\left\{u_{1}, u_{2}\right\}\right\|=\left(\left\|u_{1}\right\|^{2}+\left\|u_{2}\right\|^{2}\right)^{1 / 2}, \quad\left\{u_{1}, u_{2}\right\} \in X .
$$

Let $H=H^{n}\left(J_{1}, r_{1}\right) \times H^{m}\left(J_{2}, r_{2}\right)$ be the cartesian product Banach space.

ASSUMPTION 1. Let $J_{1}=[a, b]$ and $J_{2}-[b, c],-\infty<a<b<c<+\infty$. Let $\tau_{1}=\frac{1}{r_{1}} \sum_{k=0}^{n} P_{k} D^{k}$ and $\tau_{2}=\frac{1}{r_{2}} \sum_{k=0}^{m} Q_{k} D^{k}$, be two formal differential expressions, where $P_{k} \in C^{k}\left(J_{1}\right), k=0,1, \ldots, n, P_{n}(x) \approx 0$ on $J_{1} ; Q_{k} \in C^{k}\left(J_{2}\right), k=0,1, \ldots, m, Q_{m}(x) \neq 0$ on $J_{2} ;$ and $r_{1}(x) \in C^{0}\left(J_{1}\right)$ and $r_{2}(x) \in C^{0}\left(J_{2}\right)$ are positive real valued functions. We also assume $n \geq m$.

ASSUMPTION 2. Let $A$ and $B$ be $m \times n$ and $m \times m$ matrices with complex entries, respectively such that the range of $A=$ range of $B$, and hence, rank of $A=\operatorname{rank}$ of $B=m$.

In Section 1, we shall collect together a few definitions and results, from our earlier papers, which we require here. In Section 2, we shall present a lemma regarding the form of solutions of a type of initial value problems (IVPs) associated with the pair $\left(\tau_{1}, \tau_{2}\right)$, in terms of Green's matrices. In Section 3, we shall present an algorithm for the computation of Green's matrices for the BVPs associated with the pair $\left(\tau_{1}, \tau_{2}\right)$. In Section 4 , we shall construct the Green's matrices for problems encountered in the studies of acoustic wave guides in ocean and transverse vibrations in nonhomogeneous strings.

\section{PRELIMINARIES}

Let $f$ be a complex valued function defined on $J$. Let $f_{i}=f / J_{i}, i=1,2$. Let $J=J_{1} \cup J_{2}$. Consider

$$
\left(\tau_{1}, \tau_{2}\right) u=f
$$

and the corresponding homogeneous equation

$$
\left(\tau_{1}, \tau_{2}\right) u=0 .
$$

DEFINITION 1. We call a complex valued function $u(x)$ defined on the interval $J$, a solution (nonexplicitly mixed) of (2.1) if

(i) the functions $u / J_{1}=u_{1} \in A C^{n}\left(J_{1}\right)$ and $u / J_{2}=u_{2} \in A C^{m}\left(J_{2}\right)$ 
(ii) $\quad u_{1}$ and $u_{2}$ satisfy the equations $\tau_{1} u_{1}=f_{1}$, for $x \in J_{1}$ a.e., and $\tau_{2} u_{2}-f_{2}$, for $x \in J_{2}$ a.e., respecniveily.

DEFINITION 2. We call a complex valued function $u(x)$ defined on the interval $J$, a continuous solution of (2.1) if

(i) $\quad u$ is a solution of (2.1) in the sense of Definition 1 , and

(ii) the functions $u_{1}$ and $u_{2}$ satisfy the continuity conditions at the interface point $x=b$, namely,

$$
u_{1}^{(j)}(b-)=u_{2}^{(j)}(b+), \quad j=0,1, \ldots, m-1 .
$$

DEFINITION 3. We call a complex valued function $u(x)$ defined on the interval $J$, a matching solution of (2.1) if

(i) $\quad u$ is a solution of (2.1) in the sense of Definition 1 , and

(ii) the functions $u_{1}$ and $u_{2}$ satisfy certain matching conditions at the interface point $x-b$, namely, $A \tilde{u}_{1}(b)=B \tilde{\tilde{u}}_{2}(b)$, where

$$
\bar{u}_{1}(b)=\operatorname{column}\left(u_{1}(b), u_{1}^{(1)}(b), \ldots, u_{1}^{(n-1)}(b)\right),
$$

and

$$
\tilde{\tilde{u}}_{2}(b)=\operatorname{column}\left(u_{2}(b), u_{2}^{(1)}(b), \ldots, u_{2}^{(m-1)}(b)\right) .
$$

REMARK 1. All the above definitions can be carried over to equation (2.2) also.

Below, we recall a few definitions from [6], in the form, required here. Let $\tau=\left(\tau_{1}, \tau_{2}\right)$.

DEFINITION 5. The nonexplicitly mixed operator $N(\tau)$ is defined by

$$
\begin{aligned}
D(N(\tau)) & =\left\{\left\{u_{1}, u_{2}\right\} \in H / B_{i}^{N}\left(\left\{u_{1}, u_{2}\right\}\right)=0, i=1, \ldots, n+m\right\}, \\
N(\tau)\left\{u_{1}, u_{2}\right\} & =\left\{\tau_{1} u_{1}, \tau_{2} u_{2}\right\},
\end{aligned}
$$

where

$$
B_{i}^{N}\left(\left\{u_{1}, u_{2}\right\}\right)=\sum_{j=0}^{n-1}\left(\alpha_{i j} u_{1}^{(j)}(a)+\beta_{i j} u_{1}^{(j)}(b)\right)+\sum_{j=0}^{m-1}\left(\gamma_{i j} u_{2}^{(j)}(b)+\delta_{i j} u_{2}^{(j)}(c)\right), \quad i=1, \ldots, n+m
$$

are the linearly independent nonexplicitly mixed boundary values.

DEFINITION 6. The continuously mixed operator $C(\tau)$ is defined by

$$
D(C(\tau))=\left\{\left\{u_{1}, u_{2}\right\} \in H / B_{i}^{C}\left(\left\{u_{1}, u_{2}\right\}\right)=0, i=1, \ldots, n, u_{1}^{(j)}(b)=u_{2}(j)(b), i=1, \ldots, n\right\},
$$

$\left.c(\tau)\left\{u_{1}, u_{2}\right\}=\left\{\tau_{1} u_{1}, \tau_{2} u_{2}\right\}\right)$ where

$$
B_{i}^{C}\left(\left\{u_{1}, u_{2}\right\}\right)=\sum_{j=0}^{N-1}\left(\alpha_{i j} u_{1}^{(j)}(a)+\beta_{i j} u_{1}^{(j)}(b)+\delta_{i j} u_{2}^{(j)}(c)\right) \quad i=1, \ldots, n,
$$

are the linearly independent continuously mixed boundary values.

DEFINITION 7. The matchingly mixed operator $M(\tau)$ is defined by

$$
\begin{aligned}
D(M(\tau)) & =\left\{\left\{u_{1}, u_{2}\right\} \in H / B_{i}^{M}\left(\left\{u_{1}, u_{2}\right\}\right)=0, i=1, \ldots, n+m, A \tilde{u}_{1}(b)=B \tilde{\tilde{u}}_{2}(b),\right\}, \\
M(\tau)\left\{u_{1}, u_{2}\right\} & =\left\{\tau_{1} u_{1}, \tau_{2} u_{2}\right\},
\end{aligned}
$$

where

$$
B_{i}^{M}\left(\left\{u_{1}, u_{2}\right\}\right)=\sum_{j=0}^{N-1}\left(\alpha_{i j} u_{1}^{(j)}(a)+\delta_{i j} u_{2}^{(j)}(c)\right)+\beta_{i} \cdot \tilde{u}(b) \quad i=1, \ldots, n,
$$

are the linearly independent matchingly mixed boundary values. 
REMARK 2. For the sake of brevity, we shall study only the operators $N(\tau)$ and $M(\tau)$ and the results for the operator $C(\tau)$ follow by taking $A=B=I$ (the $n \times n$ identity matrix) in the results for $M(\tau)$.

ASSUMPTION 3. For the matchingly mixed case we assume that $n=m$.

\section{LEMMA REGARDING THE IVPS ASSOCIATED WITH $\left(\tau_{1}, \tau_{2}\right)$}

We consider a particular type of initial value problem associated with $\left(\tau_{1}, \tau_{2}\right)$ for nonexplicitly mixed and matchingly mixed operators and give a result about the form of the solution of the IVPs, in terms of Green's matrices.

(I) Nonexplicitly Mixed Initial Value Problems

Let $u_{11}, \ldots, u_{n 1}$ and $u_{12}, \ldots, u_{m 2}$ be functions in $H\left(J_{1}, r_{1}\right)$ and $H^{m}\left(J_{2}, r_{2}\right)$ which form bases for the solution spaces of $\tau_{1} u_{1}=0$ and $\tau_{2} u_{2}=0$, respectively. Then, the pairs $\left\{u_{11}, 0\right\}$, $\left\{u_{21}, 0\right\}, \ldots,\left\{u_{n 1}, 0\right\},\left\{0, u_{12}\right\}, \ldots,\left\{0, u_{m 2}\right\}$ (all of which belong to $H$ ) form basis for the solution space of $N(\tau)\left\{u_{1}, u_{2}\right\}=0$ (for the explicit form of the basis see [3]).

Define $N^{0}(\tau)$ to be the operator in $H$ such that

$$
\begin{gathered}
N^{0}(\tau)=\left\{\left\{u_{1}, u_{2}\right\} \in H / u_{1}^{(j)}(a)=0, j=0, \ldots, n-1, u_{2}^{(j)}(b)=0, j=0, \ldots m-1\right\}, \\
N^{0}(\tau)\left\{u_{1}, u_{2}\right\}=\left\{\tau_{1} u_{1}, \tau_{2} u_{2}\right\} .
\end{gathered}
$$

REMARK 3. We note that the Wronskian of $u_{11}, \ldots, u_{n 1}$, namely, $W\left(u_{11}, \ldots, u_{n 1}\right)(s)-0$ for all $s \in J_{1}$, and the Wronskian of $u_{21}, \ldots, u_{m 2}$, namely $W\left(u_{21}, \ldots, u_{m 2}\right)(s)=0$ for all $s \in J_{2}$. And, we denote by $W_{i}\left(u_{11}, \ldots, u_{n 1}\right)(s)$ the determinant obtained by replacing the $i^{\text {th }}$ column in the corresponding Wronskian by $(0,0, \ldots, 1) \in C^{n}, i-1, \ldots, n$. Similarly, we define $W_{i}\left(u_{12}, \ldots, u_{m 2}\right)(s)$.

\section{(II) Matchingly mixed initial value problems}

Let the set of pairs $\left\{u_{11}, u_{12}\right\}, \ldots,\left\{u_{n 1}, u_{n 2}\right\}$ be a basis for the solution space of $M_{0}(\tau)\left\{u_{1}, u_{2}\right\}=0$, where

$$
\begin{gathered}
M_{0}(\tau)=\left\{\left\{u_{1}, u_{2}\right\} \in H / A \tilde{u}_{1}(b)=B u_{2}(b)\right\}, \\
M_{0}(\tau)\left\{u_{1}, u_{2}\right\}=\left\{\tau_{1} u_{1}, \tau_{2} u_{2}\right\} .
\end{gathered}
$$

Also, define $M^{0}(\tau)$ to be the operator in $H$ such that

$$
\begin{gathered}
M^{0}(\tau)=\left\{\left\{u_{1}, u_{2}\right\} \in H / u_{1}^{(j)}(a)=0, j=0, \ldots, n-1, A \tilde{u}_{1}(b)=B u_{2}(b)\right\}, \\
M^{0}(\tau)\left\{u_{1}, u_{2}\right\}=\left\{\tau_{1} u_{1}, \tau_{2} u_{2}\right\} .
\end{gathered}
$$

The lemma below follows from the variation of parameters formula.

LEMMA. (I) The solution $\left\{u_{1}, u_{2}\right\}$ of $N^{0}(\tau)\left\{u_{1}, u_{2}\right\}=\left\{f_{1}, f_{2}\right\}$ is of the form $u(x)=\left\{u_{1}(x), u_{2}(x)\right\}$

$$
= \begin{cases}\int_{a}^{x} G_{11}^{o N}(x, s) f_{1}(s) r_{1}(s) d s, & x \in J_{1} \\ \int_{b}^{x} G_{22}^{a N}(x, s) f_{2}(s) r_{2}(s) d s, & x \in J_{2}\end{cases}
$$

where 


$$
\begin{aligned}
& G_{11}^{o N}(x, s)=\sum_{i=1}^{n} \frac{w_{1}\left(u_{11}, \ldots \ldots, u_{n 1}\right)(s)}{P_{n}(s) W\left(u_{11}, \ldots, u_{n 1}\right)(s)} u_{i 1}(x), \quad a<s<x<b, \\
& G_{22}^{o N}(x, s)=\sum_{i=1}^{m} \frac{W_{i}\left(u_{12}, \ldots \ldots, u_{m 2}\right)(s)}{Q_{m}(s) W\left(u_{12}, \ldots, u_{m 2}\right)(s)} u_{i 2}(x), \quad b<s<x<c,
\end{aligned}
$$

Also, we define

$$
G^{o N}=\left[\begin{array}{cc}
G_{11}^{o N} & 0 \\
0 & G_{22}^{o N}
\end{array}\right]
$$

and call $G^{o N}$ as the Green's matrix for $N^{o}(\tau)$.

II) The solution $\left\{u_{1}, u_{2}\right\}$ of $M^{o}(\tau)\left\{u_{1}, u_{2}\right\}=\left\{f_{1}, f_{2}\right\}$ is of the form $u(x)=\left\{u_{1}(x), u_{2}(x)\right\}$

$$
=\left\{\begin{array}{l}
\int_{a}^{x} G_{11}^{o M}(x, s) f_{1}(s) r_{1}(s) d s, \quad x \in J_{1} \\
\int_{a}^{b} G_{12}^{o M}(x, s) f_{1}(s) r_{1}(s) d s+\int_{b}^{x} G_{22}^{o M}(x, s) f_{2}(s) r_{2}(s) d s, \quad x \in J_{2}
\end{array}\right.
$$

where

$$
\begin{aligned}
& G_{11}^{o M}(x, s)=\sum_{i=1}^{n} \frac{W_{i}\left(u_{11}, \ldots \ldots, u_{n 1}\right)(s)}{P_{n}(s) W\left(u_{11}, \ldots, u_{n 1}\right)(s)} u_{i 1}(x), \quad a<s<x<b, \\
& G_{21}^{o M}(x, s)=\sum_{i=1}^{n} \frac{W_{i}\left(u_{11}, \ldots \ldots, u_{n 1}\right)(s)}{P_{n}(s) W\left(u_{11}, \ldots, u_{n 1}\right)(s)} u_{i 2}(x), \quad a<s<b, \quad b<x<c,
\end{aligned}
$$

and

$$
G_{22}^{a M}(x, s)=\sum_{i=1}^{m} \frac{W_{i}\left(u_{12}, \ldots \ldots, u_{m 2}\right)(s)}{Q_{m}(s) W\left(u_{12}, \ldots, u_{m 2}\right)(s)} u_{i 2}(x), \quad b<s<x<c,
$$

Also, we define

$$
G^{o M}=\left[\begin{array}{cc}
G_{11}^{o M} & 0 \\
G_{21}^{o M} & G_{22}^{o M}
\end{array}\right]
$$

and call $G^{o M}$ as the Green's matrix for $M^{\circ}(\tau)$.

\section{COMPUTATIONAL ALGORITHM FOR THE GREEN'S MATRICES FOR OPERATORS ASSOCIATED WITH $\left(\tau_{1}, \tau_{2}\right)$}

In this section, proceeding along the lines of [5], we present an algorithm for the computation of Green's matrices for operators associated with $\left(\tau_{1}, \tau_{2}\right)$.

(I) Nonexplicitly mixed operator: Consider $\left\{f_{1}, f_{2}\right\} \in X$.

Let $u(x)=\left\{u_{1}(x), u_{2}(x)\right\}=\left(N^{o}(\tau)\right)^{-1}\left\{f_{1}, f_{2}\right\}$. Then (see [4]), $u(x)=\left\{u_{1}(x), u_{2}(x)\right\}$

$$
= \begin{cases}\int_{a}^{b} G_{11}^{N}(x, s) f_{1}(s) r_{1}(s) d s+\int_{b}^{c} G_{12}^{N}(x, s) f_{1}(s) r_{1}(s) d s, & x \in J_{1} \\ \int_{a}^{b} G_{21}^{N}(x, s) f_{1}(s) r_{1}(s) d s+\int_{b}^{c} G_{22}^{N}(x, s) f_{2}(s) r_{2}(s) d s, & x \in J_{2} .\end{cases}
$$

we denote 


$$
G^{N}=\left[\begin{array}{ll}
G_{11}^{N} & G_{12}^{N} \\
G_{21}^{N} & G_{22}^{N}
\end{array}\right]
$$

and we call $G^{N}$ the Green's matrix for the operator $N(\tau)$. Let $v(x)=\left\{v_{1}(x), v_{2}(x)\right\}$ $=\left(N^{o}(\tau)\right)^{-1}\left\{f_{1}(x), f_{2}(x)\right\}$. By Theorem $4[1]$, we have $\eta(N(\tau))=n+m$. Since, $\left\{u_{1}-v_{1}, u_{2}-v_{2}\right\}$ belongs to the solution space of $\tau\left\{u_{1}, u_{2}\right\}=0$, there exists scalars $c_{1}, \ldots, c_{n+m}$ such that $u(x)=\left\{u_{1}(x), u_{2}(x)\right\}$,

$$
=\left\{\sum_{i=1}^{n} c_{i} u_{i 1}(x)+v_{1}(x), \sum_{i=1}^{m} c_{n+i} u_{i 2}(x)+v_{2}(x)\right\}
$$

Applying the boundary value on (3.3), we have $B_{l}^{N}\left(\left\{u_{1}, u_{2}\right\}\right)=0 \quad l=1,2, \ldots, n+m$. That is,

$$
B_{l}^{N}\left(\left\{\sum_{i=1}^{n} c_{i} u_{i 1}(x), \sum_{i=1}^{m} c_{n+i} u_{i 2}(x)\right\}\right)=-B_{l}^{N}\left(\left\{v_{1}, v_{2}\right\}\right)
$$

But,

$$
B_{l}^{N}\left(\left\{\sum_{i=1}^{n} c_{i} u_{i 1}(x), \sum_{i=1}^{m} c_{n+i} u_{t 2}(x)\right\}\right)-\sum_{i=1}^{n} c_{i} B_{l i}^{1}+\sum_{i=1}^{m} c_{n+i} B_{l i}^{2}
$$

where

$$
B_{l i}^{1}=\sum_{l=1}^{n-1}\left(\alpha_{j l} u_{i 1}^{(j)}(a)+\beta_{j l} u_{i 1}^{(j)}(b)\right) \quad i=1, \ldots, n
$$

and

$$
B_{l i}^{2}=\sum_{l=1}^{m-1}\left(\gamma_{j l} u_{i 1}^{(j)}(a)+\delta_{j l} u_{i 1}^{(j)}(b), \quad i=1, \ldots, m, \quad l=1, \ldots, n+m\right.
$$

Relation (3.4) can now be written as,

$$
\sum_{i=1}^{n} c_{i} B_{l i}^{1}+\sum_{i=1}^{m} c_{n+i} B_{l i}^{2}=-B_{l}^{N}\left(\left\{v_{1}, v_{2}\right\}\right), \quad l=1, \ldots, n+m .
$$

It can be verified that the coefficient matrix of the $(n+m) \times(n+m)$ linear system $(3.5)$ in $(n+m)$ unknowns, is nonsingular. Now, by the choice of $\left\{v_{1}, v_{2}\right\}$, we have

$$
\begin{aligned}
B_{l}^{N}\left(\left\{v_{1}, v_{2}\right\}\right) & =\sum_{k=0}^{n-1} \beta_{k l} v_{i 1}^{(k)}(b)+\sum_{k=0}^{m-1} \delta_{j l} v_{i 1}^{(k)}(c) \\
& =\int_{a}^{b} \mathscr{H}_{l 1}(s) f_{1}(s) r_{1}(s) d s+\int_{b}^{c} \mathscr{H}_{l 2}(s) f_{2}(s) r_{2}(s) d s
\end{aligned}
$$

where

$$
\mathscr{H}_{l 1}(s)-\sum_{j=1}^{n} \sum_{k=0}^{n-1} \frac{W_{j}\left(u_{11}, \ldots, u_{n 1}\right)(s)}{P_{n}(s) W\left(u_{11}, \ldots, u_{n 1}\right)(s)}\left(\beta_{l k} u_{l k}^{(k)}(b)\right)
$$

and

$$
\mathscr{H}_{l 2}(s)=\sum_{j=1}^{m} \sum_{k=0}^{m-1} \frac{W_{j}\left(u_{12}, \ldots, u_{m 2}\right)(s)}{Q_{m}(s) W\left(u_{12}, \ldots, u_{m 2}\right)(s)}\left(\delta_{l k} u_{l 2}^{(k)}(c)\right)
$$

$l=1, \ldots, n+m$. Clearly, $\mathscr{H}_{l 1} \in H^{n}\left(J_{1}, r_{1}\right)$ and $\mathscr{H}_{l 2} \in H^{m}\left(J_{2}, r_{2}\right)$. Rewriting (3.5), we have, for $l=1, \ldots, n+m$,

$$
\sum_{i=1}^{n} c_{i} B_{l i}^{1}+\sum_{i=1}^{m} c_{n+i} B_{l i}^{2}=-\int_{a}^{b} \mathscr{H}_{l 1}(s) f_{1}(s) r_{1}(s) d s-\int_{b}^{c} \mathscr{H}_{l 2}(s) f_{2}(s) r_{2}(s) d s .
$$


Let $B^{1}=\left(B_{l l}^{1}\right), i=1, \ldots, n$ and $B^{2}=\left(B_{l}^{2}\right), j=0, \ldots, m l=1, \ldots, n+m$. Let $B-\left[B^{1}, B^{2}\right]$. It can be shown that $B$ is a nonsingular matrix. That is, $\operatorname{det} B=0$. Consider the $(n+m) \times(n+m)$ linear system, for $l-1, \ldots, n+m$,

$$
\sum_{i=1}^{n} B_{l i}^{1}\left\{z_{i 1}, z_{i 2}\right\}+\sum_{i=1}^{m} B_{l i}^{2}\left\{z_{(n+i) 1}, z_{(n+i) 2}\right\}=-\left\{\mathcal{H}_{l 1}, \mathcal{H}_{l 2}\right\}
$$

We have by Cramer's rule, $\left\{z_{i 1}(s), z_{i 2}(s)\right\}=\frac{-1}{d e t B}\left\{B_{i 1}, B_{j 2}\right\}, i=1, \ldots, n$, and $j-1,2, \ldots, m$, where $B_{i 1}$ and $B_{j 2}$ are determinants obtained by replacing the $i^{i h}$ and $j^{\text {th }}$ columns in $B_{l i}^{1}$ and $B_{l j}^{2}$, by the column vectors $\left(\mathcal{H}_{11}, \ldots, \mathcal{H}_{(n+m) 1}\right)$ and $\left(\mathcal{H}_{12}, \ldots, \mathcal{H}_{(n+m) 2}\right)$, respectively. That is, each of $z_{i 1}(s)$ and $z_{i 2}(s)$ are linear combinations of $\mathscr{H}_{i 1}(s)$ and $\mathscr{H}_{i 2}(s)$, respectively. Hence, $\left\{z_{i 1}(s), z_{i 2}(s)\right\} \in H$. Then, we have by taking the inner-product of both sides of (3.7) with $\left\{f_{1}, f_{2}\right\}$,

$$
\begin{aligned}
\sum_{i=1}^{n} B_{l i}^{1}\left\langle\left\{z_{i 1}, z_{i 2}\right\},\left\{f_{1}, f_{2}\right\}\right\rangle+\sum_{i=1}^{m} B_{l i}^{2}\left\langle\left\{z_{(n+1) i}, z_{(n+i)}\right\},\left\{f_{1}, f_{2}\right\}\right\rangle \\
=-\left\langle\left\{\mathscr{H}_{i 1}, \mathscr{H}_{i 2}\right\},\left\{f_{1}, f_{2}\right\}\right\rangle \\
\quad-\sum_{i=1}^{n} c_{i} B_{l i}^{1}+\sum_{i=1}^{m} c_{n+i} B_{l i}^{2},
\end{aligned}
$$

(by (3.6)), which implies that,

$$
c_{i}=\left\langle\left\{z_{i 1}(s), z_{i 2}(s)\right\},\left\{f_{1}(s), f_{2}(s)\right\}\right\rangle, \quad i=1, \ldots, n+m .
$$

Combining (3.3) and (3.8), and comparing with (5), we get,

$$
\begin{aligned}
& G_{11}^{o N}(x, s)= \begin{cases}\sum_{i=1}^{n} u_{i 1}(x) z_{i 1}(s)+G_{11}^{o N}(x, s), & a<x<s<b \\
\sum_{i=1}^{n} u_{i 1}(x) z_{i 1}(s) & , a<x<s<b\end{cases} \\
& G_{12}^{o N}(x, s)=\sum_{i=1}^{n} u_{i l}(x) z_{i 1}(s), \quad a<x<b, \quad b<s<c \\
& G_{21}^{o N}(x, s)=\sum_{i=1}^{m} u_{i 2}(x) z_{i 2}(s), \quad b<x<c, a<s<b \\
& G_{22}^{o N}(x, s)= \begin{cases}\sum_{i=1}^{m} u_{i 2}(x) z_{i 2}(s)+G_{22}^{o N}(x, s), & b<s<x<c \\
\sum_{i=1}^{m} u_{i 2}(x) z_{i 2}(s) & , b<x<s<c\end{cases}
\end{aligned}
$$

This completes the algorithm for the computation of Green's matrix $G^{N}$ for the nonexplicitly mixed operator $N \tau)$.

REMARK 4. The algorithm for the computation of the Green's matrix $G^{M}$ for the operator $M(\tau)$, runs along the similar lines, with $n=m$.

\section{PHYSICAL EXAMPLES}

In this section, we shall use the computational algorithms developed in Section 3, to compute the Green's matrices for a matchingly mixed operator and a continuously mixed operator, encountered in the studies of acoustic waveguides in oceans and transverse vibrations in nonhomogeneous strings, respectively. 


\section{(I) Acoustic waveguides in oceans [6]:}

Consider the ocean to be consisting of two homogeneous layers, with a rigid bottom and a pressure release surface. Then, the propagation of acoustic waveguides in such an ocean is governed by the following equations.

$$
\begin{array}{ll}
\tau_{1} u_{1}=u_{1}^{(2)}+K_{1}^{2} u_{1}=\lambda u_{1}, & 0<x<d_{1} \\
\tau_{2} u_{2}=u_{2}^{(2)}+K_{2}^{2} u_{2}=\lambda u_{2}, & d_{1}<x<d_{2}
\end{array}
$$

together with the mixed boundary conditions given by,

$$
u_{1}(0)=u_{2}^{(1)}\left(d_{2}\right)=0, \quad u_{1}\left(d_{1}\right)=u_{2}\left(d_{2}\right), \quad \frac{1}{\rho_{1}} u_{1}\left(d_{1}\right)=\frac{1}{\rho_{2}} u_{2}\left(d_{1}\right),
$$

where $\rho_{1}$ and $\rho_{2}$ are constant densities of the two layers, $K_{1}, K_{2}$ are constants which depend upon the frequency constant $\omega$ and the constant sound velocities $c_{1}, c_{2}$ of the two layers, respectively, $\lambda$ is an unknown constant, $\left[0, d_{1}\right]$ and $\left[d_{1}, d_{2}\right]$ denote the depth of the two layers and $u_{1}$ and $u_{2}$ stand for the depth eigenfunctions. Let $J_{1}=\left[0, d_{1}\right]$ and $J_{2}-\left[d_{1}, d_{2}\right]$. The matching conditions at the interface $x=d_{1}$ can be written in the matrix form $A_{1} \bar{u}_{1}\left(d_{1}\right)=B_{2} \tilde{u}_{2}\left(d_{1}\right)$, where $\tilde{u}_{i}\left(d_{1}\right)=\operatorname{column}\left(u_{i}\left(d_{1}\right), u_{i}^{(1)}\left(d_{1}\right)\right)$, $A_{i}=\left(\begin{array}{cc}1 & 0 \\ 0 & 1 / \rho_{i}\end{array}\right)$ for $i=1,2$. Also, we have $n=m=d=2$. Define

$$
\begin{aligned}
M(\tau) & =\left\{\left\{u_{1}, u_{2}\right\} \in H^{2}\left(J_{1}, 1 / \rho_{1}\right) \times H^{2}\left(J_{2}, 1 / \rho_{2}\right) / A_{1} \tilde{u}_{1}\left(d_{1}\right)=A_{1} \tilde{u}_{2}\left(d_{1}\right), u_{1}(0)=u_{2}^{(1)}\left(d_{2}\right)=0\right\}, \\
M(\tau) u & =\left\{\tau_{1} u_{1}, \tau_{2} u_{2}\right\} .
\end{aligned}
$$

After simple calculations along lines of the algorithm, we get the form of the Green's matrix $G^{M}$ to be of the form,

$G_{11}^{M}= \begin{cases}\frac{\sin K_{1} x}{K_{1} M}\left(\rho_{2} K_{1} \cos K_{2}\left(d_{2}-d_{1}\right) \cos K_{1}\left(d_{1}-s\right)-\rho_{1} K_{2} \sin K_{1}\left(d_{1}-s\right) \sin K_{2}\left(d_{2}-d_{1}\right)\right), & 0<x<s<d_{1}, \\ \frac{\sin K_{1} s}{K_{1} M}\left(\rho_{2} K_{1} \cos K_{2}\left(d_{2}-d_{1}\right) \cos K_{1}\left(d_{1}-x\right)-\rho_{1} K_{2} \sin K_{1}\left(d_{1}-x\right) \sin K_{2}\left(d_{2}-d_{1}\right)\right), & 0<s<x<d_{1},\end{cases}$

$G_{12}^{M}=\frac{\rho_{1}}{M} \sin K_{1} x \cos K_{2}\left(d_{2}-s\right), \quad 0<x<d_{1}, \quad d_{1}<s<d_{2}$

$G_{12}^{M}=\frac{\rho_{2}}{M} \sin K_{1} s \cos K_{2}\left(d_{2}-x\right), \quad 0<s<d_{1}, \quad d_{1}<x<d_{2}$

$G_{11}^{M}=\left\{\begin{array}{l}\frac{\cos K_{2}\left(d_{2}-s\right)}{K_{1} M}\left(\rho_{2} K_{1} \cos K_{2}\left(d_{2}-d_{1}\right) \cos K_{1}\left(d_{1}-s\right)-\rho_{2} K_{1} \cos K_{1} d_{1} \sin K_{2}\left(x-d_{1}\right)\right), \quad d<x<s<d, \\ \frac{\cos K_{2}\left(d_{2}-x\right)}{K_{2} M}\left(\rho_{2} K_{2} \sin K_{1} d_{1} \cos K_{2}\left(d_{1}-s\right)+\rho_{2} K_{1} \cos K_{1} d \sin K_{2}\left(s-d_{1}\right)\right), \quad d_{1}<s<x<d_{2},\end{array}\right.$

We also note that

$$
A_{1} \tilde{G}_{11}^{M}\left(d_{1}, s\right)=A_{2} \tilde{G}_{21}^{M}\left(d_{1}, s\right)
$$

and

$$
A_{1} \tilde{G}_{12}^{M}\left(d_{1}, s\right)=A_{2} \tilde{G}_{22}^{M}\left(d_{1}, s\right)
$$

REMARK 5. In the above, we have the compact and general form of the Green's matrix of the problem compared to the one given in [6]. 
(II) Transverse vibrations in nonhomogeneous strings [7]:

Consider the string consisting of two portions of lengths $d_{1}$ and $d_{2}-d_{1}$, and different uniform densities $\rho_{1}, \rho_{2}$ respectively, having tension $T$ and stretched between the points $x-0$ and $x-d_{2}$. The modes of transverse vibrations of the above string are governed by,

$$
\tau_{1} u_{1}=c_{1}^{2}\left(-u_{1}^{(2)}\right)=\lambda u_{1}, \quad 0<x<d_{1},
$$

and

$$
\tau_{2} u_{2}=c_{2}^{2}\left(-u_{2}^{(2)}\right)=\lambda u_{2}, \quad d_{1}<x<d_{2},
$$

together with the mixed boundary conditions given by,

$$
u_{1}(0)=u_{2}\left(d_{2}\right)=0, \quad u_{1}\left(d_{1}\right)=u_{2}\left(d_{1}\right), \quad u_{1}^{(1)}\left(d_{1}\right)=u_{2}^{(1)}\left(d_{1}\right),
$$

where $c_{i}^{2}=T / \rho_{i}, i=1,2$. Here, the conditions at the interface point are the continuity conditions.

Proceeding along the lines of the algorithm, we get, after routine calculations, the Green's matrix $G$ to be of the form,

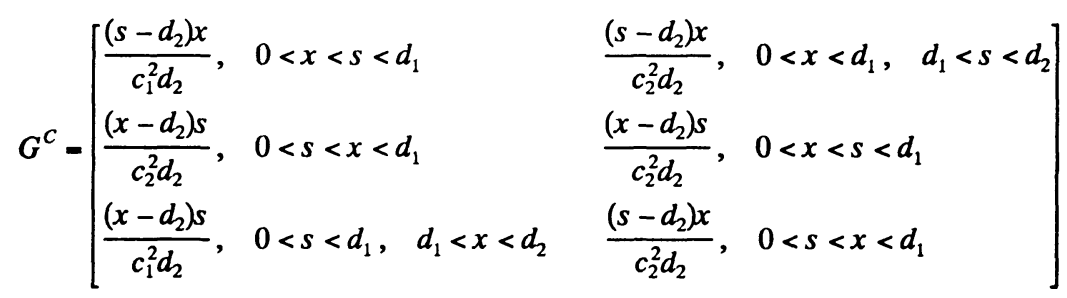

We note that $\tilde{G}_{11}^{c}\left(d_{1}, s\right)=\tilde{G}_{21}^{C}\left(d_{1}, s\right)$ and similar relations are true of the components $G_{12}^{C}$ and $G_{22}^{C}$.

\section{ACKNOWLEDGMENT}

The authors dedicate the work to the chancellor of the institute Bhagavan sri sathya sai baba.

\section{REFERENCES}

[1] VENKATESULU, $M$. and BHASKAR, T. GNANA, Solutions of initial value problems associated with a pair of mixed linear ordinary differential equations, J. Math. Anal. Appl. 146 (2), 1991, 63-78.

[2] VENKATESULU, M. and BHASKAR, T. QNANA, Fundamental systems and solutions of nonhomogeneous equations associated with a pair of mixed linear ordinary differential equations, J. Aust. Math. Soc, series A49, 1990.

[3] VENKATESULU, M. and BHASKAR, T. GNANA, Selfadjoint boundary value problems associated with a pair of mixed linear ordinary differential equations, J. Math. Anal. Appl. 144 (2), 1989.

[4] VENKATESULU, M. and BHASKAR, T. GNANA, Green's matrices for boundary value problems associated with a pair of mixed linear regular ordinary differential operators, to appear in Zeitschrift f. Anal.

[5] LOCKER, JOHN, Functional analysis and two point differential operators, Pitman Research Notes, 1986.

[6] BOYLES, C. ALLAN, Acoustic Waveguides, Applications to Oceanic Sciences, Wiley, New York, 1984.

[7] GHOSH, P. K. The Mathematics of Waves and Vibrations, Macmillan, Delhi, India, 1975. 


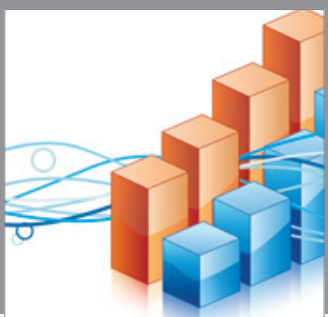

Advances in

Operations Research

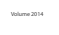

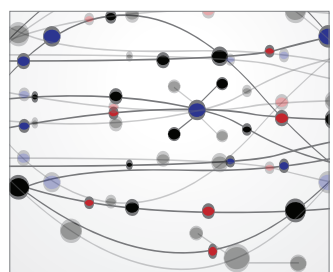

\section{The Scientific} World Journal
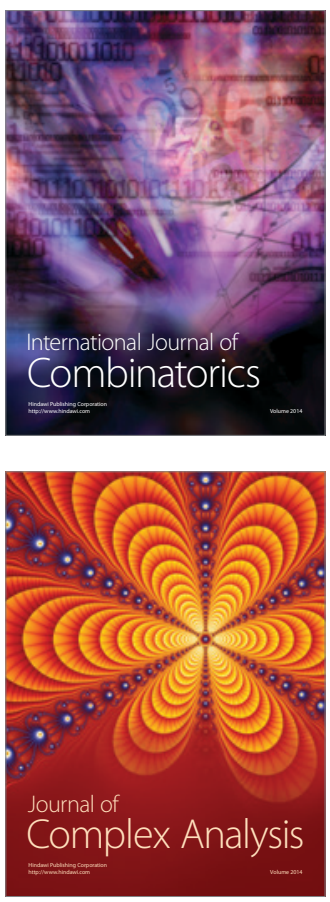

International Journal of

Mathematics and

Mathematical

Sciences
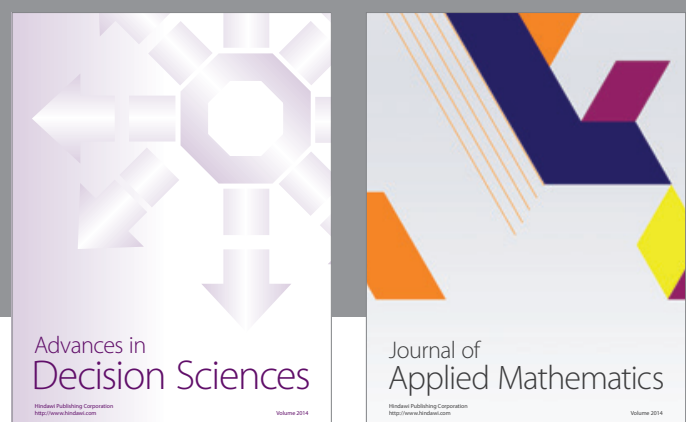

Journal of

Applied Mathematics
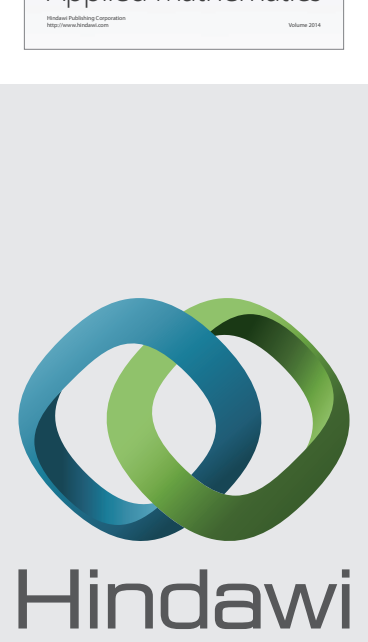

Submit your manuscripts at http://www.hindawi.com
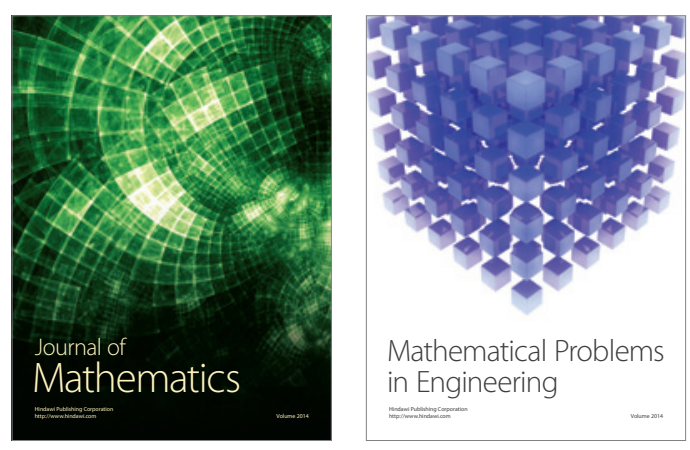

Mathematical Problems in Engineering
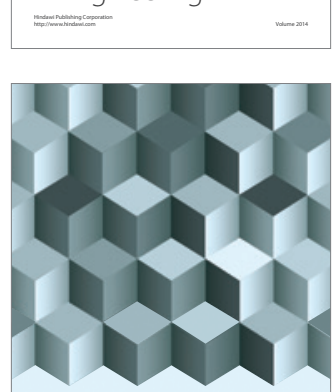

Journal of

Function Spaces
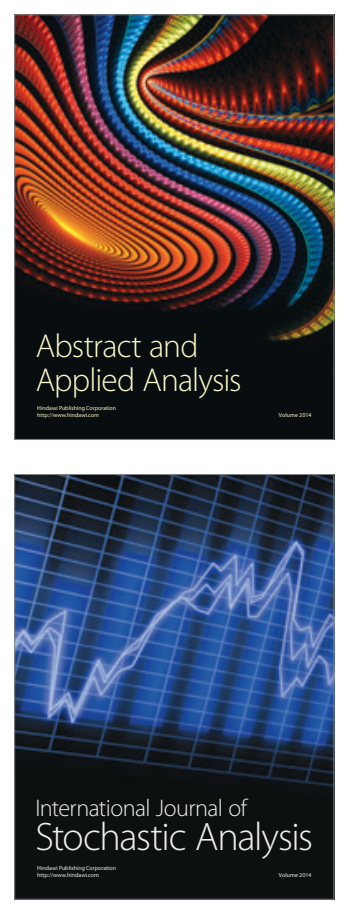

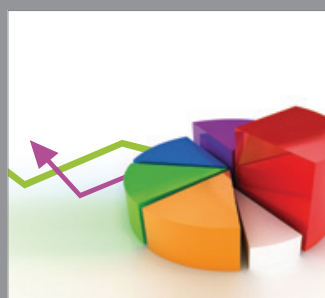

ournal of

Probability and Statistics

Promensencen
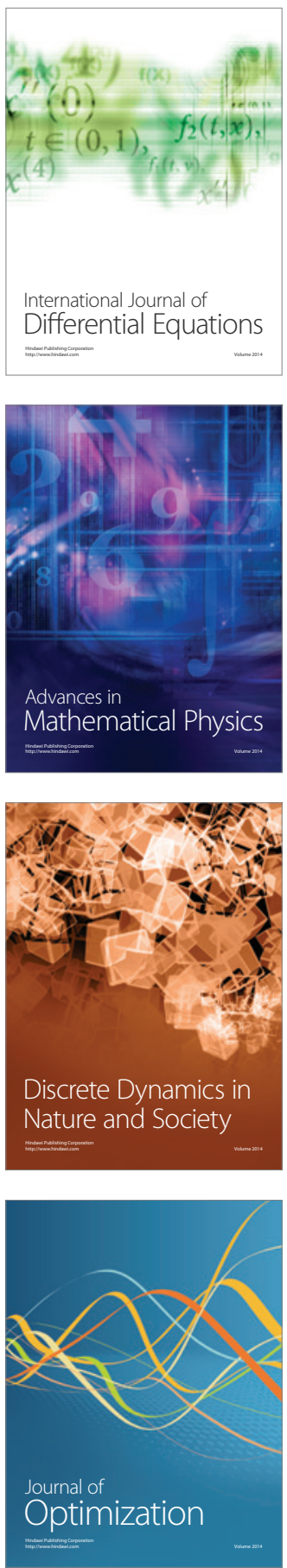